\title{
ON THE UNIQUENESS FOR SUB-CRITICAL QUASI-GEOSTROPHIC EQUATIONS*
}

\author{
LUCAS C. F. FERREIRA ${ }^{\dagger}$
}

\begin{abstract}
We prove uniqueness of mild solutions in the class $C\left([0, T) ; L^{\frac{n}{2 \gamma-1}}\right), 0<T \leq \infty$, for sub-critical quasi-geostrophic equations without assuming any smallness condition. As a consequence, any mild solution in $C\left([0, \infty) ; L^{\frac{2}{2 \gamma-1}}\right)$ satisfies the regularity and decay properties given in the previous paper [4]. The proof is performed in the framework of Lorentz spaces.
\end{abstract}

Key words. Quasi-geostrophic equations, uniqueness, large time behavior, Lorentz space.

AMS subject classifications. 35Q35, 35A05, 76D03, 35B40, 35D10, 42B35, 86A10.

\section{Introduction}

In this work we are concerned with the initial value problem (IVP) for the quasigeostrophic equation:

$$
\begin{cases}\frac{\partial \theta}{\partial t}+u \nabla_{x} \theta+(-\Delta)^{\gamma} \theta=0, & x \in \mathbb{R}^{n}, t>0, \\ \theta(x, 0)=\theta_{0}(x), & x \in \mathbb{R}^{n},\end{cases}
$$

where $n \geq 1$ and $\frac{1}{2}<\gamma<\frac{n+2}{4}$. The velocity field $u$ is determined from the normalized temperature $\theta$ through a linear operator $\mathcal{R}[\theta]=u$, such that $\nabla \cdot u=0$ and

$$
u_{j}=\sum_{i=1}^{n} a_{i j} \mathcal{R}_{i}(\theta), \text { for } 1 \leq j \leq n,
$$

where $\mathcal{R}_{i}=\partial_{i}(-\Delta)^{-\frac{1}{2}}$ is the $i$-th Riesz transform and the $a_{i j}$ 's are constants. By means of the Duhamel principle, the IVP (1.1) is converted to the integral equation

$$
\theta(t)=G_{\gamma}(t) \theta_{0}-B(\theta, \theta)(t)
$$

where

$$
B(\theta, \psi)(t)=\int_{0}^{t} \nabla_{x} G_{\gamma}(t-s)(\psi \mathcal{R}[\theta])(s) d s
$$

and $G_{\gamma}(t)$ is the convolution operator with kernel $g_{\gamma}$ given by $\widehat{g_{\gamma}}(\xi, t)=e^{-|\xi|^{2 \gamma} t}$. Throughout the present paper, a solution of (1.3) will be called a mild solution for the IVP (1.1).

The physical case $n=2$ and $u=\left(-\mathcal{R}_{2}(\theta), \mathcal{R}_{1}(\theta)\right)$ corresponds to well known 2D surface quasi-geostrophic equations with fractional dissipation (2DQG) which have been used in models of geophysical fluid dynamics. They are derived from general quasi-geostrophic equations in the special case of small Rossby number and vertically stratified flows [5, 13].

*Received: October 9, 2009; accepted (in revised version): April 25, 2010. Communicated by Paul Milewski.

${ }^{\dagger}$ Universidade Estadual de Campinas, Departamento de Matemática, CEP 13083-859, CampinasSP, Brazil (lcff@ime.unicamp.br). L.C.F. Ferreira was partially supported by project CNPQ 305542/2009-5, Brazil. 
The theory of geophysical dynamics has a large number of applications, which has motivated several authors to study 2DQG from a mathematical point of view; see e.g. $[2,5,6,7,8,9,10,12,14]$ and their references. Concerning the uniqueness of LerayHopf weak solutions, some results were obtained in $[6,8,9]$ by assuming further LerayProdi-Serrin or integral-regularity type restrictions. Later on, in a remarkable paper, the authors of [2] show that Leray-Hopf weak solutions with arbitrary data $\theta_{0} \in L^{2}\left(\mathbb{R}^{2}\right)$ and $1 / 2 \leq \gamma \leq 1$ become smooth for any $t>0$, and as a consequence those solutions are unique. Furthermore, it follows that smooth solutions do not develop singularities at finite time. For periodic conditions, this last breakthrough also was proved in [10] by a different method based on the preservation of a certain continuity modulus. In [7] this approach was extended for the whole space setting. In the supercritical case $0 \leq \gamma<\frac{1}{2}$, it is still an open problem to know whether smooth solutions blow up (or not) at finite time (see $[10])$.

In $[6,12,14]$ the authors proved some interesting asymptotic results, which, for given data $\theta_{0} \in L^{2}$, assure the existence of one Leray-Hopf weak solution satisfying certain decays. In the case $\frac{1}{2} \leq \gamma \leq 1$, any weak solution presents the decays given in $[6,12,14]$ because the uniqueness holds true in that range.

On the other hand, the authors of [3] proved well-posedness of small mild solutions for (1.1) in the framework of weak- $L^{p}$ spaces, and thereby they obtained existence of solutions in $B C\left([0, \infty) ; L^{\frac{n}{2 \gamma-1}}\right)$. Later on, the same authors obtained in [4] some decay rates and asymptotic behavior results in Lebesgue spaces for solutions of 2DQG and all their derivatives. In particular, without assuming any smallness condition, they showed the existence of a global mild solution $\theta \in C\left([0, \infty) ; L^{\frac{n}{2 \gamma-1}}\right)$ and uniqueness in the class $C\left([0, \infty) ; L^{\frac{n}{2 \gamma-1}}\right) \cap C\left((0, \infty) ; L^{q}\right)$ with $q>\frac{n}{2 \gamma-1}$. Among other decays, that solution satisfies

$$
\lim _{t \rightarrow \infty} t^{\frac{|k|}{2 \gamma}}\left\|\nabla_{x}^{k} \theta(\cdot, t)\right\|_{L^{\frac{n}{2 \gamma-1}}}=0 \text { and } \lim _{t \rightarrow \infty} t^{\frac{|k|}{2 \gamma}+\alpha_{q}}\left\|\nabla_{x}^{k} \theta(\cdot, t)\right\|_{L^{q}}=0
$$

for every multi-index $k$ and $q>\frac{n}{2 \gamma-1}$, where $\alpha_{q}=\frac{2 \gamma-1}{2 \gamma}-\frac{n}{2 \gamma q}$. As well as for the case of Leray-Hopf weak solutions, it is natural to wonder whether any mild solution belonging to $C\left([0, \infty) ; L^{\frac{n}{2 \gamma-1}}\right)$ presents the same large time behavior (1.5). Motivated by this, it emerges the need to prove the uniqueness of solutions in the class $C\left([0, \infty) ; L^{\frac{n}{2 \gamma-1}}\right)$. The aim of the present paper is to show this property by employing the framework of weak- $L^{p}$ spaces. Precisely, we prove the following result:

THEOREM 1.1. Assume $\frac{1}{2}<\gamma<\frac{n+2}{4}$ and $0<T \leq \infty$. If $\theta$ and $\psi$ are two mild solutions of (1.1) in $C\left([0, T) ; L^{\frac{n}{2 \gamma-1}}\right)$ with the same initial data $\theta_{0}$, then $\theta(\cdot, t)=\psi(\cdot, t)$ for all $t \in[0, T)$. Consequently, for $n=2$ any mild solution in $C\left([0, \infty) ; L^{\frac{n}{2 \gamma-1}}\right)$ satisfies the property (1.5).

Let us recall that a Leray-Hopf weak solution for $(1.1)$ with data $\theta_{0} \in L^{2}$ is a solution in the sense of distributions that belongs to $L^{\infty}\left((0, T) ; L^{2}\right) \cap L^{2}\left((0, T) ; H^{\gamma}\right)$. Since $L^{\frac{2}{2 \gamma-1}}\left(\mathbb{R}^{2}\right) \nsubseteq L^{2}\left(\mathbb{R}^{2}\right)$ when $\gamma \neq 1$, clearly a mild solution in $C\left([0, T) ; L^{\frac{2}{2 \gamma-1}}\right)$ is not in general a Leray-Hopf weak solution (in particular, the data are taken in different classes). Thus, beyond employing a distinct approach, our uniqueness result (even for $n=2$ ) is different from the above-mentioned result for Leray-Hopf weak solutions. Also, we remark that the decay property (1.5) is obtained by assuming just $\theta_{0} \in L^{\frac{2}{2 \gamma-1}}$.

Finally, we refer the reader to [11] for uniqueness results in spirit of Theorem 1.1 for Navier-Stokes equations $n \geq 3$. The paper is organized as follows: In Section 2, for the sake of completeness, we recall some properties about Lorentz spaces, and in Section 3 we prove Theorem 1.1. 


\section{Lorentz spaces}

In this section we recall some properties about Lorentz spaces. For a deeper discussion we refer the reader to [1]. The distribution function of a measurable function $f$ is defined by $\lambda_{f}(s)=m\left(\left\{x \in \mathbb{R}^{n}:|f(x)|>s\right\}\right)$, with $m$ standing for the Lebesgue measure on $\mathbb{R}^{n}$. The Lorentz spaces $L^{(p, q)}$ is the set of all measurable functions such that the norm $\|\cdot\|_{(p, q)}$

$$
\|f\|_{(p, q)}= \begin{cases}\left(\frac{p}{q} \int_{0}^{\infty}\left[t^{\frac{1}{p}} f^{* *}(t)\right]^{q} \frac{d t}{t}\right)^{\frac{1}{q}}, & \text { if } 1<p<\infty, 1 \leq q<\infty, \\ \sup _{t>0} t^{\frac{1}{p}} f^{* *}(t), & \text { if } 1<p \leq \infty, q=\infty,\end{cases}
$$

is finite, where

$$
f^{* *}(t)=\frac{1}{t} \int_{0}^{t} f^{*}(s) d s \text { and } f^{*}(t)=\inf \left\{s>0: \lambda_{f}(s) \leq t\right\} .
$$

Equivalently, one can define $L^{(p, q)}$ (including for $0<p \leq 1$ ) by means of the quantity $\|\cdot\|_{(p, q)}^{*}$ given by $(2.1)$ with $f^{*}$ in place of $f^{* *}$. One has $L^{(p, p)}=L^{p}$, and $L^{(p, \infty)}$ is also called weak- $L^{p}$ space. This space is the largest one among $L^{(p, q)}$-spaces, since the continuous inclusions

$$
L^{(p, 1)} \subset L^{\left(p, q_{1}\right)} \subset L^{p} \subset L^{\left(p, q_{2}\right)} \subset L^{(p, \infty)}
$$

hold true for all $1 \leq q_{1} \leq p \leq q_{2} \leq \infty$. The dual space of $L^{(p, 1)}$ is $L^{\left(p^{\prime}, \infty\right)}$ and the one of $L^{(p, q)}$ is $L^{\left(p^{\prime}, q^{\prime}\right)}$ for $1<p, q<\infty$. Moreover, interpolation theory in Lorentz spaces yields

$$
\left(L^{\left(p_{0}, q_{0}\right)}, L^{\left(p_{1}, q_{1}\right)}\right)_{\theta, q}=L^{(p, q)},
$$

provided that $1<p_{0}<p_{1}<\infty, 0<\theta<1, \frac{1}{p}=\frac{1-\theta}{p_{0}}+\frac{\theta}{p_{1}}$ and $1 \leq q_{0}, q_{1}, q \leq \infty$. The property (2.2) still holds true for $0<p_{0} \leq 1$, but in this case one needs to consider $L^{\left(p_{0}, q_{0}\right)}$ endowed with the quantity $\|\cdot\|_{(p, q)}^{*}$ instead of $\|\cdot\|_{(p, q)}$. Hölder-type inequalities work well in Lorentz spaces, namely

$$
\|h\|_{(r, s)} \leq C(r)\|f\|_{\left(p_{1}, q_{1}\right)}\|g\|_{\left(p_{2}, q_{2}\right)},
$$

for $1<p_{1}, p_{2}<\infty, \frac{1}{r}=\frac{1}{p_{1}}+\frac{1}{p_{2}}, s \geq 1$ and $\frac{1}{q_{1}}+\frac{1}{q_{2}} \geq \frac{1}{s}$.

\section{Proof of the uniqueness result}

First we recall some properties for $\left\{G_{\gamma}(t)\right\}_{t \geq 0}$ on the Lorentz spaces.

Lemma 3.1. Assume that $0<\gamma<\infty, 1 \leq p_{1} \leq p_{2} \leq \infty, 1 \leq q_{1}, q_{2} \leq \infty$ and $k \in(\{0\} \cup$ $\mathbb{N})^{n}$ is a multi-index. Then

$$
\left\|\nabla_{x}^{k} G_{\gamma}(t) \theta_{0}\right\|_{\left(p_{2}, q_{2}\right)} \leq C t^{-\frac{|k|}{2 \gamma}-\frac{n}{2 \gamma}\left(\frac{1}{p_{1}}-\frac{1}{p_{2}}\right)}\left\|\theta_{0}\right\|_{\left(p_{1}, q_{1}\right)} .
$$

Moreover, if $\theta_{0} \in L^{\frac{n}{2 \gamma-1}}, \frac{1}{2}<\gamma \leq \frac{n+1}{2}, \frac{n}{2 \gamma-1}<q \leq \infty$, and $\alpha_{q}=\frac{2 \gamma-1}{2 \gamma}-\frac{n}{2 \gamma q}$, then

$$
\lim _{t \rightarrow 0^{+}}\left\|G_{\gamma}(t) \theta_{0}-\theta_{0}\right\|_{L^{\frac{n}{2 \gamma-1}}}=0 \text { and } \lim _{t \rightarrow 0^{+}} t^{\alpha_{q}}\left\|G_{\gamma}(t) \theta_{0}\right\|_{L^{q}}=0 .
$$


Proof. The property (3.2) is proved in [4, Lemma 2.1], and (3.1) follows by interpolating the inequality (2.1) of [4].

The estimates below can be found in [3] (see also [15] for (3.3) in the case $\gamma=1$ and $n \geq 3$ ), but we include their proofs for the convenience of the reader.

Lemma 3.2. Suppose that $0<\gamma<\infty$ and $1<p<q<\infty$. Then

$$
\int_{0}^{\infty} t^{\frac{1}{2 \gamma}\left(\frac{n}{p}-\frac{n}{q}-(2 y-1)\right)}\left\|\nabla_{x} G_{\gamma}(t) \phi\right\|_{(q, 1)} d s \leq C\|\phi\|_{(p, 1)} .
$$

Moreover, if $n>2(2 \gamma-1)>0$ then

$$
\sup _{t>0}\|B(\theta, \psi)\|_{\left(\frac{n}{2 \gamma-1}, \infty\right)} \leq K \sup _{t>0}\|\theta(\cdot, t)\|_{\left(\frac{n}{(2 \gamma-1)}, \infty\right)} \sup _{t>0}\|\psi(\cdot, t)\|_{\left(\frac{n}{(2 \gamma-1)}, \infty\right)} .
$$

Proof. Let $g(t)=t^{\frac{1}{2 \gamma}\left(\frac{n}{p}-\frac{n}{q}-(2 \gamma-1)\right)}\left\|\nabla_{x} G_{\gamma}(t) \phi\right\|_{(q, 1)}$ and $1<p_{1}<p<p_{2}<q$ such that $\left(\frac{n}{p}-\frac{n}{p_{2}}\right)<2 \gamma$. By Lemma 3.1, one has

$$
g(t) \leq C t^{\frac{n}{2 \gamma}\left(\frac{1}{p}-\frac{1}{p_{k}}\right)-1}\|\phi\|_{\left(p_{k}, 1\right)}, \text { for } k=1,2 .
$$

Take $0<l_{2}<1<l_{1}$ satisfying $\frac{1}{l_{k}}=\frac{n}{2 \gamma}\left(\frac{1}{p_{k}}-\frac{1}{p}\right)+1$. Notice that (3.5) implies $g(t) \in$ $L^{l_{k}, \infty}(0, \infty)$ and $\|g(t)\|_{L^{\left(l_{k}, \infty\right)}((0, \infty))} \leq C\|\phi\|_{\left(p_{k}, 1\right)}$ for $k=1,2$. Interpolation theorems in Lorentz spaces [1] yield

$$
\|g(t)\|_{L^{1}(0, \infty)} \leq C\|\phi\|_{(p, 1)},
$$

which is equivalent to (3.3). In order to treat (3.4), we define

$$
f(\cdot, s)=(\psi \mathcal{R}[\theta])(\cdot, t-s) \text { if } 0<s<t \text { and } f(\cdot, s)=0 \text { otherwise. }
$$

Next observe that $\frac{2 \gamma-1}{n}=1-\frac{n-(2 \gamma-1)}{n}$ and $\frac{n}{n-2(2 \gamma-1)}>\frac{n}{n-(2 \gamma-1)}>1$. Thus, from duality and Hölder's inequality (2.3), we have

$$
\begin{aligned}
& \|B(\theta, \psi)\|_{\left(\frac{n}{2 \gamma-1}, \infty\right)}=\sup _{\|\phi\|_{\left(\frac{n}{n-(2 \gamma-1)}, 1\right)}}\left|\int_{\mathbb{R}^{n}} B(\theta, \psi) \phi(x) d x\right| \\
& =\sup _{\|\phi\|\left(\frac{n}{n-(2 \gamma-1)}, 1\right)}|=| \int_{\mathbb{R}^{n}} \int_{0}^{\infty}\left(\nabla_{x} G_{\gamma}(\cdot, s) * f(\cdot, s)\right)(x) d s \phi(x) d x \mid
\end{aligned}
$$

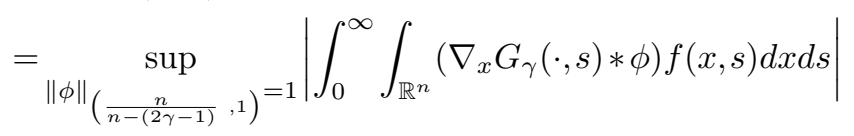

$$
\begin{aligned}
& \leq_{\|\phi\|} \sup _{\left(\frac{n}{n-(2 \gamma-1)}, 1\right)} \int_{0}^{\infty}\|f(\cdot, s)\|_{\left(\frac{n}{2(2 \gamma-1)}, \infty\right)}\left\|\nabla_{x} G_{\gamma}(\cdot, s) * \phi\right\|_{\left(\frac{n}{n-2(2 \gamma-1)}, 1\right)} d s .
\end{aligned}
$$

Finally, since $\frac{1}{2 \gamma}\left(\frac{n}{p}-\frac{n}{q}-(2 y-1)\right)=0$ when $p=\frac{n}{n-(2 \gamma-1)}$ and $q=\frac{n}{n-2(2 \gamma-1)}$, the inequality (3.3), together with Hölder's inequality and the $L^{(p, \infty)}$-continuity of Riesz transforms, allow us to estimate the right hand side of (3.6) by 


$$
\begin{aligned}
& \leq C \sup _{t>0}\|f(\cdot, t)\|_{\left(\frac{n}{2(2 \gamma-1)}, \infty\right)}\left\|\sup _{\left(\frac{n}{n-(2 \gamma-1)}, 1\right)}\right\|=1 \\
& \leq K \|_{\left(\frac{n}{n-(2 \gamma-1)}, 1\right)} \\
& \leq \sup _{t>0}\|\theta(\cdot, t)\|_{\left(\frac{n}{(2 \gamma-1)}, \infty\right)} \sup _{t>0}\|\psi(\cdot, t)\|_{\left(\frac{n}{(2 \gamma-1)}, \infty\right)} .
\end{aligned}
$$

\subsection{Proof of Theorem 1.1.}

First step. Let us denote $\omega=\theta-\psi, \phi=G_{\gamma}(t) \theta_{0}-\theta$ and $\zeta=G_{\gamma}(t) \theta_{0}-\psi$, and for simplicity $p_{\gamma}=\frac{n}{2 \gamma-1}$. In this step we show that $\omega(\cdot, t)=0$ in $[0, \widetilde{T}]$, for some $\widetilde{T}>0$ small enough. To begin, we estimate the difference $\omega$ as

$$
\begin{aligned}
\|B(\theta, \theta)-B(\psi, \psi)\|_{\left(p_{\gamma}, \infty\right)} & =\|B(\theta, \theta)-B(\psi, \theta)+B(\psi, \theta)-B(\psi, \psi)\|_{\left(p_{\gamma}, \infty\right)} . \\
& \leq\|B(\omega, \theta)\|_{\left(p_{\gamma}, \infty\right)}+\|B(\psi, \omega)\|_{\left(p_{\gamma}, \infty\right)} .
\end{aligned}
$$

Writing $\theta$ and $\psi$ in terms of $\phi$ and $\zeta$, respectively, and afterwards inserting them into (3.7), we can bound the right hand side of (3.7) by

$$
\begin{aligned}
& \leq\|B(\omega, \phi)\|_{\left(p_{\gamma}, \infty\right)}+\left\|B\left(\omega, G_{\gamma}(t) \theta_{0}\right)\right\|_{\left(p_{\gamma}, \infty\right)}+\|B(\zeta, \omega)\|_{\left(p_{\gamma}, \infty\right)}+\left\|B\left(G_{\gamma}(t) \theta_{0}, \omega\right)\right\|_{\left(p_{\gamma}, \infty\right)} \\
& :=I_{1}(t)+I_{2}(t)+I_{3}(t)+I_{4}(t) .
\end{aligned}
$$

The inequality (3.4) implies, for all $t \in[0, \widetilde{T}]$, that

$$
I_{1} \leq C \sup _{0<t<\widetilde{T}}\|\omega\|_{\left(p_{\gamma}, \infty\right)} \sup _{0<t<\widetilde{T}}\|\phi\|_{\left(p_{\gamma}, \infty\right)} \text { and } I_{3} \leq C \sup _{0<t<\widetilde{T}}\|\omega\|_{\left(p_{\gamma}, \infty\right)} \sup _{0<t<\widetilde{T}}\|\zeta\|_{\left(p_{\gamma}, \infty\right)}
$$

In order to deal with $I_{2}$ and $I_{4}$, take $\frac{1}{l}=\frac{1}{p_{\gamma}}+\frac{1}{d}$ and apply (3.1) with $p_{2}=p_{\gamma}$ and $p_{1}=l$ to obtain

$$
\begin{aligned}
I_{2}+I_{4} & \leq 2 C \int_{0}^{t}(t-s)^{-\frac{n}{2 \gamma}\left(\frac{1}{l}-\frac{1}{p_{\gamma}}\right)-\frac{1}{2 \gamma}}\|\omega(\cdot, s)\|_{\left(p_{\gamma}, \infty\right)}\left\|G_{\gamma}(t) \theta_{0}\right\|_{(d, \infty)} d s \\
& \leq C \sup _{0<t<\widetilde{T}}\|\omega(\cdot, t)\|_{\left(p_{\gamma}, \infty\right)}\left(\sup _{0<t<\widetilde{T}} t^{\alpha_{d}}\left\|G_{\gamma}(t) \theta_{0}\right\|_{(d, \infty)}\right) \int_{0}^{t}(t-s)^{-\frac{n}{2 \gamma d}-\frac{1}{2 \gamma} s^{-\alpha_{d}} d s} \\
& \leq C \sup _{0<t<\widetilde{T}}\|\omega(\cdot, t)\|_{\left(p_{\gamma}, \infty\right)}\left(\sup _{0<t<\widetilde{T}} t^{\alpha_{d}}\left\|G_{\gamma}(t) \theta_{0}\right\|_{(d, \infty)}\right),
\end{aligned}
$$

for all $t \in(0, \widetilde{T})$, where we have used above that $-\frac{n}{2 \gamma d}-\frac{1}{2 \gamma}-\alpha_{d}+1=0$ and

$$
\int_{0}^{t}(t-s)^{-\frac{n}{2 \gamma d}-\frac{1}{2 \gamma}} s^{-\alpha_{d}} d s=t^{-\frac{n}{2 \gamma d}-\frac{1}{2 \gamma}-\alpha_{d}+1} \int_{0}^{1}(1-s)^{-\frac{n}{2 \gamma d}-\frac{1}{2 \gamma}} s^{-\alpha_{d}} d s=C<\infty .
$$

Now, adding the inequalities (3.8) and (3.9) one gets

$$
\sup _{0<t<\widetilde{T}}\|\omega(\cdot, t)\|_{\left(p_{\gamma}, \infty\right)}=\sup _{0<t<\widetilde{T}}\|B(\theta, \theta)-B(\psi, \psi)\|_{\left(p_{\gamma}, \infty\right)} \leq \Gamma(\widetilde{T}) \sup _{0<t<\widetilde{T}}\|\omega(\cdot, t)\|_{\left(p_{\gamma}, \infty\right)},
$$

with $\Gamma(\widetilde{T})$ given by

$$
\begin{aligned}
\Gamma(\widetilde{T}) & =C\left(\sup _{0<t<\widetilde{T}}\|\phi(\cdot, t)\|_{\left(p_{\gamma}, \infty\right)}+\sup _{0<t<\widetilde{T}}\|\zeta(\cdot, t)\|_{\left(p_{\gamma}, \infty\right)}+\sup _{0<t<\widetilde{T}} t^{\alpha_{d}}\left\|G_{\gamma}(t) \theta_{0}\right\|_{(d, \infty)}\right) \\
& \leq C\left(\sup _{0<t<\widetilde{T}}\|\phi(\cdot, t)\|_{L^{p_{\gamma}}}+\sup _{0<t<\widetilde{T}}\|\zeta(\cdot, t)\|_{L^{p_{\gamma}}}+\sup _{0<t<\widetilde{T}} t^{\alpha_{d}}\left\|G_{\gamma}(t) \theta_{0}\right\|_{L^{d}}\right),
\end{aligned}
$$


since the continuous inclusion $L^{r} \subset L^{(r, \infty)}$ holds true. Notice that $\|\phi(\cdot, t)\|_{L^{p_{\gamma}}},\|\zeta(\cdot, t)\|_{L^{p_{\gamma}} \rightarrow 0}$ as $t \rightarrow 0^{+}$, because $\theta, \psi$ and $G_{\gamma}(t) \theta_{0}$ (see (3.2) in Lemma 3.1) take the same initial condition $\theta_{0} \in L^{p_{\gamma}}$. Thus we can choose $\widetilde{T}>0$ small enough so that $\Gamma(\widetilde{T})<1$, and then $\omega(\cdot, t)=\theta(\cdot, t)-\psi(\cdot, t)=0$ for all $t \in[0, \widetilde{T}]$.

Second step. Next we show that $\theta(\cdot, t)=\psi(\cdot, t)$ in $[0, T)$. To this end, define

$$
T^{*}=\sup \{\widetilde{T}: 0<\widetilde{T}<T, \theta(\cdot, t)=\psi(\cdot, t) \text { for all } t \in[0, \widetilde{T})\} .
$$

If $T^{*}=T$, then we are done. Otherwise, we have $0<T^{*}<\infty, \theta(\cdot, t)=\psi(\cdot, t)$ for $t \in$ $\left[0, T^{*}\right)$, and by continuity $\theta\left(\cdot, T^{*}\right)=\psi\left(\cdot, T^{*}\right)$. From the first step, there is $\sigma>0$ so small that $\theta(\cdot, t)=\psi(\cdot, t)$ for $t \in\left[T^{*}, T^{*}+\sigma\right)$. Therefore $\theta(\cdot, t)=\psi(\cdot, t)$ in $\left[0, T^{*}+\sigma\right)$, which contradicts the definition of $T^{*}$.

\section{REFERENCES}

[1] J. Bergh and J. Löfström, Interpolation Spaces, Springer, Berlin-Heidelberg-New York, 1976.

[2] L.A. Caffarelli and A. Vasseur, Drift diffusion equations with fractional diffusion and the quasigeostrophic equation, Ann. Math., 171, 1903-1930, 2010.

[3] J.A. Carrillo and L.C.F. Ferreira, Self-similar solutions and large time asymptotics for the dissipative quasi-geostrophic equation, Monatsh. Math., 151, 111-142, 2007 (preprint posted in January 2005 at www.uab.cat/matematiques).

[4] J.A. Carrillo and L.C.F. Ferreira, The asymptotic behaviour of subcritical dissipative quasigeostrophic equations, Nonlinearity, 21, 1001-1018, 2008.

[5] P. Constantin, A. Majda and E. Tabak, Formation of strong fronts in the 2D quasi-geostrophic thermal active scalar, Nonlinearity, 7, 1459-1533, 1994.

[6] P. Constantin and J. Wu, Behavior of solutions of 2D quasi-geostrophic equations, SIAM J. Math. Anal., 30, 937-948, 1999.

[7] H. Dong and D. Du, Global well-posedness and a decay estimate for the critical dissipative quasi-geostrophic equation in the whole space, Discrete Contin. Dyn. Syst., 21, 1095-1101, 2008.

[8] N. Ju, Existence and uniqueness of the solution to the dissipative 2D quasi-geostrophic equations in the sobolev space, Commun. Math. Phys., 251, 365-376, 2004.

[9] N. Ju, On the two dimensional quasi-geostrophic equations, Indiana Univ. Math. J., 54, 897926, 2005.

[10] A. Kiselev, F. Nazarov and A. Volberg, Global well-posedness for the critical 2D dissipative quasi-geostrophic equation, Invent. Math., 167, 445-453, 2007.

[11] P. Lemarié-Rieusset, Recent Developments in the Navier-Stokes Problem, Chapman \& Hall, Boca Raton, 2002.

[12] C.J. Niche and M.E. Schonbek, Decay of weak solutions to the 2D dissipative quasi-geostrophic equation, Commun. Math. Phys., 276, 93-115, 2007.

[13] J. Pedlosky, Geophysical Fluid Dynamics, Springer-Verlag, New-York, 1987.

[14] M.E. Schonbek and T.P. Schonbek, Asymptotic behavior to dissipative quasi-geostrophic flows, SIAM J. Math. Anal., 35, 357-375, 2003.

[15] M. Yamazaki, The Navier-Stokes equations in the weak- $L^{n}$ spaces with time-dependent external force, Math. Ann., 317, 635-675, 2000. 